\title{
A DIC/Moire Hybrid Method Based on Regular Pattern
}

\author{
Hao Wu, Kai Kang, and Zhiyong Wang* \\ Department of Mechanics, School of Mechanical Engineering, Tianjin University, \\ Tianjin 300072, China
}

Digital Image Correlation (DIC) is a superior tool that makes it possible to measure the surface deformation of objects with a high level of accuracy. Traditional view thinks that there must be random characteristic in the speckle pattern adopted in DIC, but in this paper the application of regular pattern in DIC method is developed and proved to have better performance than random pattern. Subsequently, if the used pattern in DIC is regular, it is proved that nonmaximum correlation coefficient includes the information of deformation. A new Moiré processing method, based on correlation coefficient, is also proposed in this study for deformation measurement directly without any optimization iterations. Finally, by combining the two methods, a DIC/Moiré hybrid method with regular pattern is used to measure large in-plane deformation. Verification experiments are conducted to prove the less computed time demand and high accuracy in our method. 